\title{
Implantable micromechanical parylene-based pressure sensors for unpowered intraocular pressure sensing
}

\author{
Po-Jui Chen ${ }^{1}$, Damien C Rodger ${ }^{2,3}$, Rajat Agrawal ${ }^{3,4}$, \\ Saloomeh Saati ${ }^{4}$, Ellis Meng ${ }^{5}$, Rohit Varma ${ }^{3,4}$, \\ Mark S Humayun ${ }^{3,4}$ and Yu-Chong Tai ${ }^{1,2}$ \\ ${ }^{1}$ Department of Electrical Engineering, California Institute of Technology, Pasadena, \\ CA 91125, USA \\ ${ }^{2}$ Department of Bioengineering, California Institute of Technology, Pasadena, CA 91125, \\ USA \\ ${ }^{3}$ Keck School of Medicine, University of Southern California, Los Angeles, CA 90033, USA \\ ${ }^{4}$ Doheny Eye Institute, Los Angeles, CA 90033, USA \\ ${ }^{5}$ Department of Biomedical Engineering, University of Southern California, Los Angeles, \\ CA 90089, USA \\ E-mail: pjchen@mems.caltech.edu
}

Received 24 June 2007, in final form 17 July 2007

Published 31 August 2007

Online at stacks.iop.org/JMM/17/1931

\begin{abstract}
This paper presents the first implantable, unpowered, parylene-based microelectromechanical system (MEMS) pressure sensor for intraocular pressure (IOP) sensing. From in situ mechanical deformation of the compliant spiral-tube structures, this sensor registers pressure variations without electrical or powered signal transduction of any kind. Micromachined high-aspect-ratio polymeric hollow tubes with different geometric layouts are implemented to obtain high-sensitivity pressure responses. An integrated device packaging method has been developed toward enabling minimally invasive suture-less needle-based implantation of the device. Both in vitro and ex vivo device characterizations have successfully demonstrated $\mathrm{mmHg}$ resolution of the pressure responses. In vivo animal experiments have also been conducted to verify the biocompatibility and functionality of the implant fixation method inside the eye. Using the proposed implantation scheme, the pressure response of the implant can be directly observed from outside the eye under visible light, with the goal of realizing convenient, direct and faithful IOP monitoring in glaucoma patients.
\end{abstract}

(Some figures in this article are in colour only in the electronic version)

\section{Introduction}

Glaucoma is a debilitating disease affecting the optic nerve that results in loss of vision for hundreds of millions of people worldwide. Studies indicate that elevated intraocular pressure (IOP) due to accumulated ocular fluid is one of the most significant risk factors for this eye disease [1]. Although IOP monitoring is known as the most effective method to evaluate the progression of glaucoma, conventional clinical techniques including contact and non-contact tonometry have difficulty in faithfully recording IOP values inside the eye, and are problematic in deployment for regular (e.g. daily) monitoring 
of IOP fluctuations. Their readouts also have insufficient precision due to the fact that current tonometers heavily depend on calculations of ocular mechanics, which have large variation due to the varied dimensions and mechanical properties of different eyes [2, 3]. These considerations together articulate the great need for a direct, convenient and reliable IOP sensing technology.

Microelectromechanical system (MEMS) is an enabling technology to fabricate miniaturized sensors, actuators and systems. Having characteristic dimensions at the microscale, MEMS devices/systems are highly suitable for implantable applications, particularly for implantable IOP sensors. Many telemetric MEMS pressure sensors, using passive or active measurement modalities, have been developed to realize continuous IOP monitoring directly from inside the eye with accurate and precise pressure readouts [4-8]. However, the sensors will eventually be implanted inside the eye and exposed to the in vivo environment such that power consumption would be a concern in the use of the active sensors. On the other hand, insufficient reading distance due to limited magnetic coupling impedes the use of the existing passive sensors for practical IOP sensing. Additionally, all these sensors were fabricated in sizes that are large compared with the human eye, which is not favorable to their implantation in terms of surgical procedures and time.

Recently, an all-mechanical unpowered pressure sensing technology has been proposed as a novel IOP measurement paradigm [9]. In essence, the device is a deformable spiraltube parylene pressure sensor that facilitates micromechanical pressure sensing without electrical circuitry or external powered signal transduction. When the sensor is implanted on the iris of the eye as shown in figure 1, its in situ mechanical pressure response corresponding to the IOP variation can be observed with an optical reader, such as a camera, a microscope or a stereoscope, from outside the eye through the transparent cornea. In the implantation scheme as shown in figure 2, the device is fabricated in a small form factor so that it can be accommodated inside a hypodermic needle, and hence minimally invasive suture-less implantation can be accomplished with a needle incision and device injection. Similarly to telemetric sensing, this optical methodology has the potential of enabling a patient to read his/her own IOP (e.g. using his/her one eye to observe the other where the

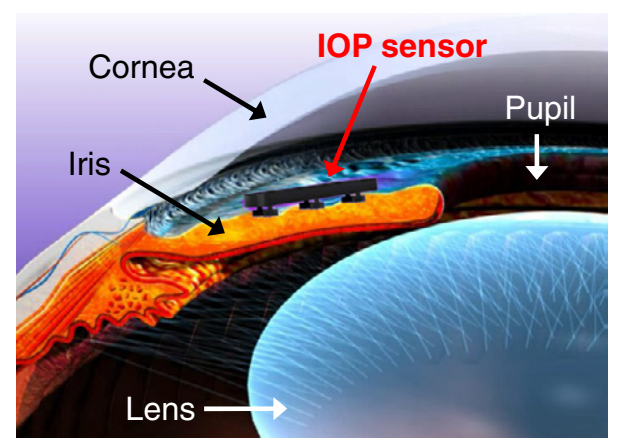

Figure 1. Ocular anatomy of anterior chamber of the eye. The pressure sensor is designed for implantation on the iris so its visible IOP response can be externally observed through the transparent cornea. Because of its specific location the device is highly sensitive to the IOP variation. sensor is implanted through a special binocular reader) instead of visiting the ophthalmologist, resulting in more practical and convenient IOP tracking.

In this work, the micromechanical spiral-tube pressure sensor has been improved in order to prove the concept of unpowered IOP sensing. Different novel geometric designs as well as state-of-the-art micromachining technologies are implemented to achieve higher pressure responses of the sensors. Two types of IOP sensors (high-sensitivity and needle-implantable) are fabricated and characterized to show the capability of detecting small pressure variations (at the $\mathrm{mmHg}$ level) and of being implanted through a needle. A monolithically integrated device packaging method is also developed so that the packaged devices are able to be securely anchored and functional after their suture-less implantation on the iris. Finally, a complete suite of in vitro and in vivo testing demonstrates the feasibility of this promising IOP sensing technology. All animal procedures in this study conformed to the ARVO Statement on the Use of Animals in Ophthalmic and Vision Research.

\section{Design}

\subsection{IOP sensor}

The IOP sensing element is a high-aspect-ratio compliant structure inspired by a Bourdon tube, a free-standing curved hollow tube [10]. It has a systematic, well-defined pressure response on its mechanical conformation. The tube deforms by applying a bending moment generated from a pressure difference applied between the pressure encapsulated in the tube/channel and the ambient pressure. The deformation, which can be visualized by movement of the pointing tip at the free end of the tube, is linearly related to this pressure difference. Therefore, the corresponding environmental (outside tube) pressure can be measured given that the pressure inside the tube is set at a predetermined constant. Because of the visible tube deformation after implantation, direct IOP measurements can be obtained through optical observation. Variation in IOP can occur due to such reasons as treatment of underlying disease, diurnal rhythm and atmospheric pressure change. All of these changes would be accurately detected by the sensor. Different geometric designs can be used to amplify the tube deformation as well as modify its in-plane deformation trajectories. Table 1 gives possible layouts of the IOP sensor design including an Archimedean spiral to increase the angular rotation at the end pointing tip, and a longarmed spiral tube to maximize the lateral movement of the end arm. These tube geometries can both enlarge the pressure sensitivity and optimize the device dimensions for sutureless clinical implantation using small outer-diameter needles. Furthermore, the minimized out-of-plane deformation using these symmetric tube designs leads to more accurate pressure readouts from such optical observation.

\subsection{Device packaging}

Because the pressure sensor is designed to be implanted inside the eye for a direct IOP measurement, packaging is necessary to ensure that the IOP sensor is fully functional after implantation. In order to avoid handling and 

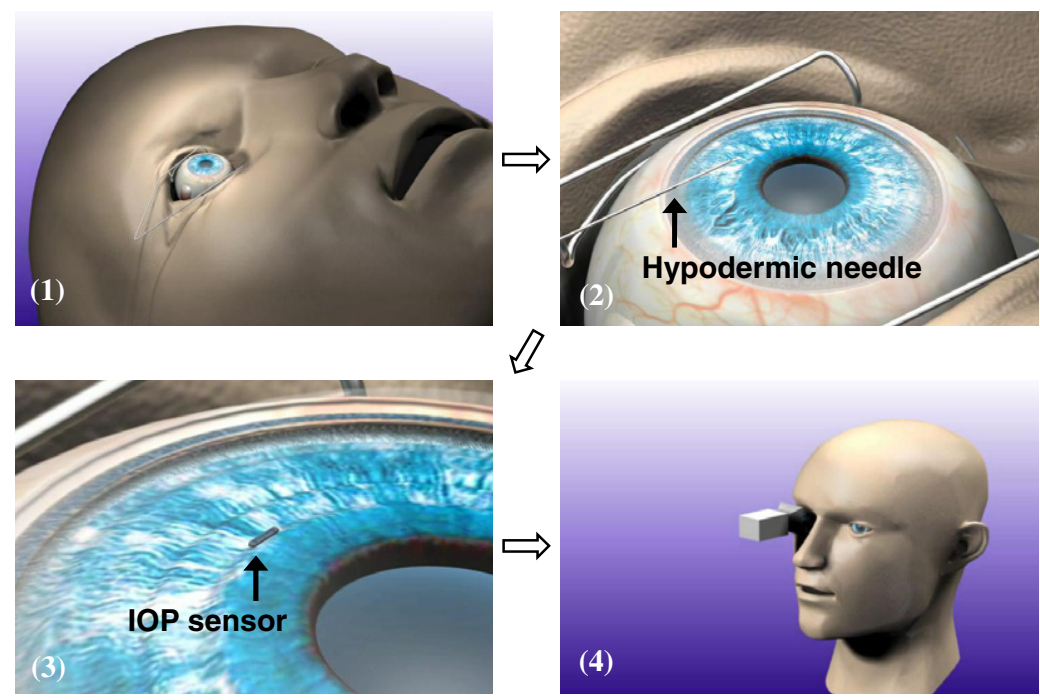

Figure 2. The proposed needle-based suture-less implantation scheme: (1) eye stabilization using ocular speculum; (2) small-gauge hypodermic needle incision of the cornea; (3) anchoring of the device on the iris after unloading from the needle and (4) IOP readouts from outside the eye by optical observation of the visible pressure response of the sensor.

Table 1. Geometric designs of compliant structures for the IOP sensor.

$\begin{aligned} & \text { Curved tube } \\ & \text { (basic Bourdon tube) }\end{aligned}$
$\begin{aligned} & \text { Spiral tube } \\ & \text { (angular deformation) }\end{aligned}$
Long-armed tube

alignment issues during assembly, a wafer-level integrated device packaging method has been developed. By implementing micromachining technology, a fully packaged device comprising the following components can be fabricated on a monolithic substrate as shown in figure 3: (1) a sensor platform to support the IOP sensor; (2) bottom posts with an end 'anchoring feet' design to realize suture-less anchoring of the implant on the iris [11]; and (3) a perforated flexible parylene membrane to protect the sensor from damage during implantation. With these features, a packaged device can be implanted on the iris so that faithful IOP readouts from the sensor are observable from outside the eye through the cornea and the protective parylene membrane. The resulting implant fixation method also obviates the use of sutures while additionally favoring the minimally invasive implantation scheme that curtails complications during and after surgery.

\subsection{Material selection}

As the main component of the IOP sensor, the thin-walled hollow tubes should be flexible to facilitate more sensitive

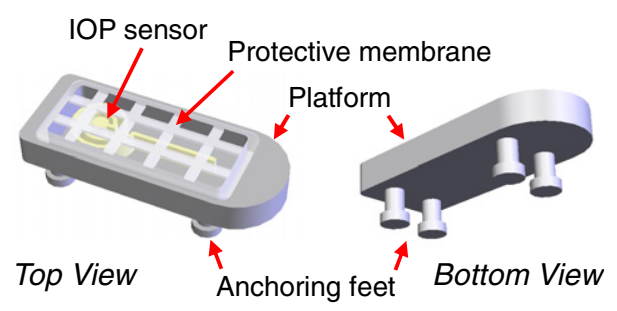

Figure 3. Schematics of a fully-packaged implantable device.

pressure responses. Conceptually, higher aspect-ratio tubes in a specific shape (e.g. elliptical or rectangular cross-section) are necessary to obtain larger in-plane deformations and stronger out-of-plane resistance to environmental interference. Parylene C (poly-para-xylene C) is selected as the structural material for the tube because of its desirable properties, including mechanical flexibility (Young's modulus $\sim 4 \mathrm{GPa}$ ), chemical inertness and biocompatibility (USP Class VI grade and ISO 10993 certified) [12, 13]. Thin-film parylene is coated using chemical vapor deposition (CVD) at room temperature in a manner compatible with CMOS/MEMS processes. More importantly, its pinhole-free conformal coating ability enables fabrication of high-aspect-ratio hollow tubes in a single material layer. Microfabricated parylenebased devices have been reported in applications such as implantable microelectrodes, neurocages, neuroprobes and retinal prostheses [14-19]. These examples support utilization of parylene for flexible and implantable biomedical microdevices.

\section{Fabrication}

Two types of IOP sensors, high-sensitivity and needleimplantable, were microfabricated. The high-sensitivity sensor is a simplified version that includes only the parylene tube, while the needle-implantable sensor has a smaller form factor and has all necessary components for implantation: the 
parylene tube, the top protective membrane and the bottom tissue anchoring structures. All sensors were microfabricated on a monolithic substrate to accomplish integrated device packaging. Figure 4 illustrates the fabrication process of the needle-implantable sensors. It started with growing a $0.75 \mu \mathrm{m}$ thick wet oxide on a $500 \mu \mathrm{m}$ thick double-side-polished silicon wafer. After patterning the backside oxide using buffered hydrofluoric acid (Transene Company Inc., Danvers, MA) and photoresist as a mask, backside two-step deep-reactive-ion etching (DRIE) in a PlasmaTherm SLR system (Unaxis Inc., St. Petersburg, FL) was performed to define the backside access holes as well as $200 \mu \mathrm{m}$ high feet anchors and trench boundaries of the sensor platform. A novel embedded channel fabrication technology was then employed to create the highaspect-ratio quasi-rectangular parylene channels [20]. With frontside oxide patterning, two-step complementary plasmabased dry etching and an $8 \mu \mathrm{m}$ thick parylene $\mathrm{C}$ deposition in a PDS system (Specialty Coating Systems Inc., Indianapolis, IN), $180 \mu \mathrm{m}$ high and $16.5 \mu \mathrm{m}$ wide thin-walled embedded channels with $5 \mu \mathrm{m}$ thick parylene walls were successfully fabricated. Channels with an internal channel aspect ratio of approximately 26 with a quasi-rectangular profile (sidewall angle $\sim 90^{\circ} \pm 1^{\circ}$ ) were obtained to achieve large mechanical

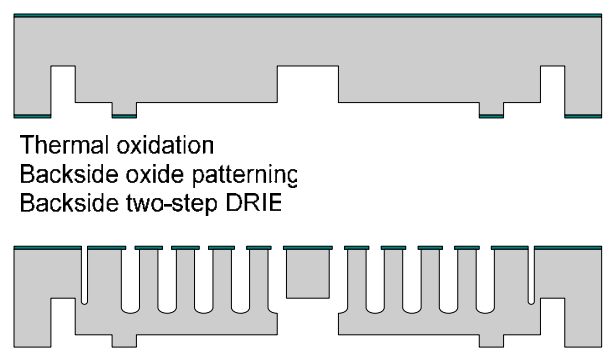

Frontside oxide patterninc

Complementary higr-aspect-ratio trench etching

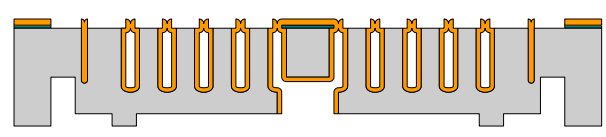

$1^{\text {s1 }}$ layer parylene deposition and patterning Oxide removal

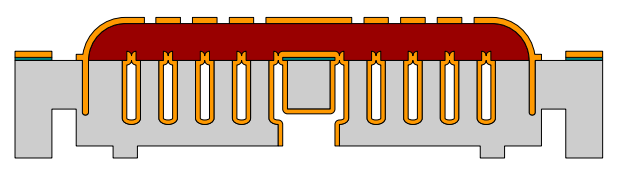

Sacrificial photoresist coating and patterning $2^{\text {nd }}$ layer parylene deposition and patterning

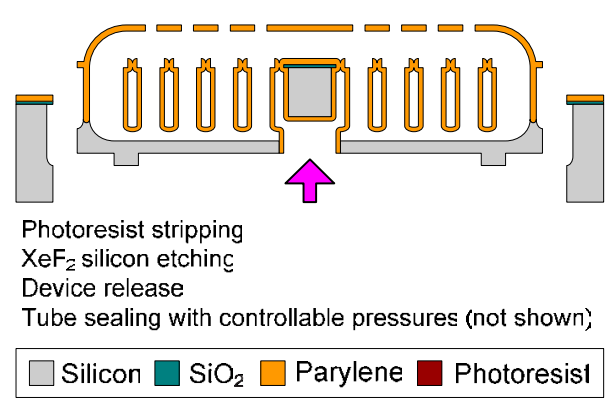

Figure 4. Process flow for device fabrication. The arrow denotes the location of final parylene tube sealing. responses. The backside holes located at the center of the tubes were also connected to the inside of the parylene channels after the frontside trench etching. These holes would be sealed after device fabrication to encapsulate air at a constant pressure inside the tubes. Oxygen plasma etching with a photoresist etch mask was performed to separate the embedded parylene channels into tube patterns with various geometric designs (spiral tube and long-armed tube described above). Subsequent to removal of the remaining frontside oxide, a sacrificial photoresist layer was spin coated and the second parylene C layer was deposited and patterned to construct a $10 \mu \mathrm{m}$ thick perforated parylene membrane caging the channels from $20 \mu \mathrm{m}$ above. Finally, the photoresist was stripped with acetone and the parylene channels were released using an isotropic $\mathrm{XeF}_{2}$ gaseous silicon etch in a customized system. Using the same through-wafer technique, the entire microdevice was separated from the wafer substrate after $\mathrm{XeF}_{2}$ etching without dicing while the main body of the device remained intact due to protection from the parylenefilled trenches depicted in figure 4. A thin layer of parylene could be further coated on the released device to promote its biocompatibility.

Figure 5 shows the high-sensitivity IOP sensors micromachined using only the parylene tube fabrication process. Sensors of both the spiral-tube and long-armedtube designs were successfully fabricated. Micromachined integrated packaging components for the needle-implantable IOP sensors before the final $\mathrm{XeF}_{2}$ device release are shown in figure 6. The embedded IOP sensors are clearly observable through the top perforated protective membranes due to the optical transparency of parylene C. Note that an excessively long leverage arm should be avoided as it has a substantial mechanical resistance which affects the rotational behavior of the spiral tube. Instead, a pair of long-armed-tube sensors could be placed in the same platform to effectively double the pressure-related movement by observing the distance between the pointing tips. This 'rotation-tip' readout amplification method is promising, but it requires delicate calibration of the residual stress in the parylene structures for good alignment of the pointing tips after the tube release [21, 22]. Compared with the spiral-tube sensors, the long-armed-tube sensors were well suited for fabrication in such a form factor that they can be accommodated inside a 19-gauge needle as shown in figure 7 while achieving a reasonable pressure response. The important physical dimensions of the microfabricated IOP sensors characterized in the following section are listed in table 2 .
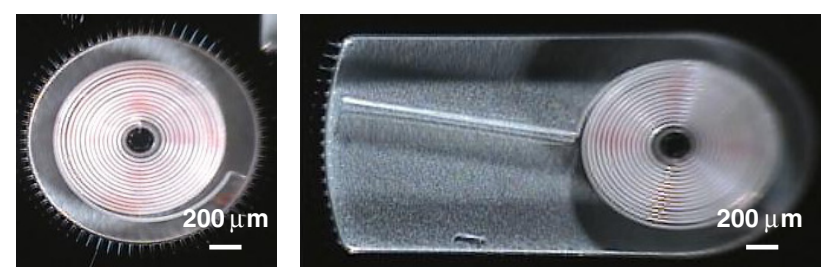

Figure 5. Microfabricated high-sensitivity parylene-based IOP sensors: (left) spiral-tube sensor and (right) long-armed-tube sensor. Note that the indicators surrounding the sensors were fabricated to accurately determine the position of the end pointing tip. 
Implantable micromechanical parylene-based pressure sensors

Table 2. Important physical dimensions of the fabricated micromechanical IOP sensors.

\begin{tabular}{lccll}
\hline & \multicolumn{2}{c}{$\begin{array}{c}\text { High-sensitivity sensors } \\
\text { (large form factor) }\end{array}$} & & $\begin{array}{c}\text { Needle-implantable sensors } \\
\text { (small form factor) }\end{array}$ \\
\cline { 2 - 3 } \cline { 5 - 5 } Tube design & Spiral tube & Long-armed tube & & Long-armed tube \\
\hline Overall size & $2 \times 2 \times 0.5 \mathrm{~mm}^{3}$ & $4 \times 2 \times 0.5 \mathrm{~mm}^{3}$ & & $3 \times 0.6 \times 0.5 \mathrm{~mm}^{3}$ \\
Spiral diameter & $\sim 1100 \mu \mathrm{m}$ & $\sim 1100 \mu \mathrm{m}$ & & $\sim 400 \mu \mathrm{m}$ \\
Spiral turns & 15 & 15 & 8 \\
Pointing tip length & $100 \mu \mathrm{m}$ & $1500 \mu \mathrm{m}$ & & $750 \mu \mathrm{m}$ \\
Indicator separation & $5^{\circ}$ & $80 \mu \mathrm{m}$ & & $20 \mu \mathrm{m}$ \\
\hline
\end{tabular}

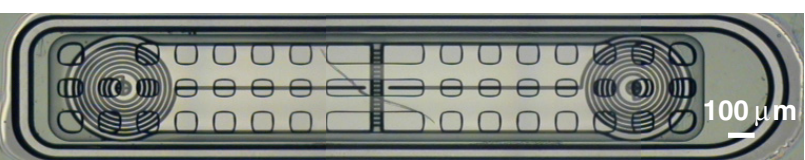

(a)

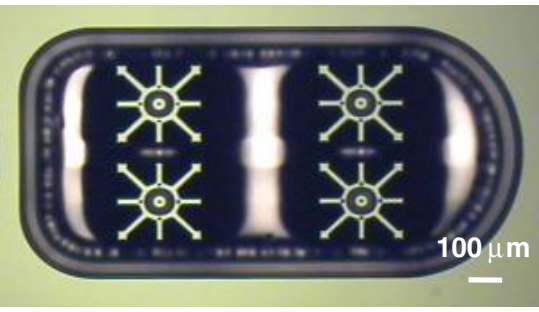

(b)

Figure 6. Integrated packaging components of the needle-implantable parylene-based IOP sensors: $(a)$ micrograph of the parylene tube in the long-armed-tube design and the top perforated parylene membrane before final device release (top view); (b) example of backside tissue anchoring structures: (left) top view; (right) side view (200 $\mu \mathrm{m}$ in height).
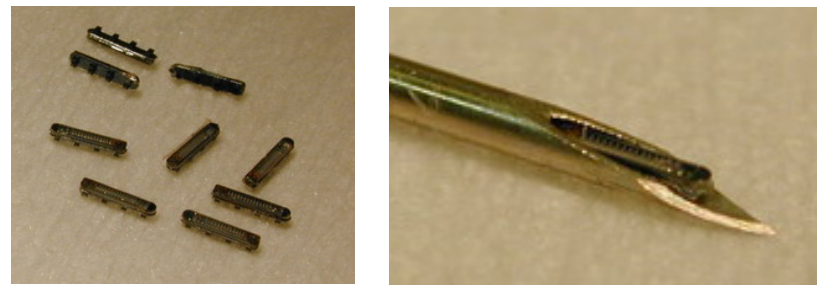

Figure 7. Microfabricated needle-implantable parylene-based IOP sensors: (left) released devices; (right) a fabricated device situated inside a 19-gauge hypodermic needle.

\section{Results and discussion}

\subsection{In vitro pressure sensor testing}

The microfabricated parylene-based pressure sensors were first tested on-bench to characterize their in vitro pressure responses. All parylene tubes were sealed by applying epoxy over the backside access holes with the atmospheric pressure ( $1 \mathrm{~atm}$ ) serving as the gauge reference pressure. A customized pressure regulation setup was used to vary the environmental pressure surrounding the sensors. The structural deformation of the sensors with respect to different positive pressures was measured using a stereoscope and compared with numerical readouts from a pressure gauge. Because the parylene tube structures are strengthened by their high-aspect-ratio shape, the sensors were not affected by stiction on their underside to the platform and were completely functional in both air and liquid media. Prior to immersion and operation in liquid, however, the sensors need to go through appropriate surface treatments such as short oxygen plasma roughening in order to reduce the severity of bubble formation on parylene surfaces [9], which can result in device failure. The pressure testing of the sensors was conducted in water to mimic the intraocular environment. For the high-sensitivity sensors, the testing results shown in figure 8 indicate that their pressure sensitivity was $0.47^{\circ} \mathrm{mmHg}^{-1}$ in the rotational displacement with the spiral-tube design and $11.23 \mu \mathrm{m} \mathrm{mmHg}^{-1}$ in the lateral displacement with the long-armed-tube design, both showing a continuous pressure response (continuous pointing tip rotation) with high linearity. These data further verify that different geometric tube layouts result in different trajectories of the tube deformation. The pressure sensing resolution was approximately $3.56 \mathrm{mmHg}$ obtained with the longarmed-tube sensors, which falls in the range of interest for IOP measurements. This resolution was determined by the confidence of observation of tip movement through half the distance between $80 \mu \mathrm{m}$ spaced indicators. Consequently, it is worth noting that, even without redesigning the parylene tube structure, higher pressure resolution can be effectively achieved using indicators with a smaller separation and optical readers with a higher magnification. This fact leaves room to further optimize the micromechanical pressure sensor. The needle-implantable sensors with long-armed-tube design also demonstrated a $3.21 \mu \mathrm{m} \mathrm{mmgg}^{-1}$ pressure sensitivity in the lateral displacement as shown in figure 9.

\subsection{In vivo anchoring scheme testing}

The micromachined tissue anchors have been studied to evaluate their in vivo efficacy under normal conditions in a rabbit eye model. A small angled razor blade incision was made in the cornea of the right eye of two rabbits. Healon viscoelastic (Advanced Medical Optics, Santa Ana, CA) was injected to maintain separation of the iris from the cornea during device insertion. The dummy silicon substrate with three anchoring feet (all components coated with approximately $1.5 \mu \mathrm{m}$ of parylene C) were implanted using forceps and gently depressed from above to embed the anchors in the iris. After retraction of the forceps, no additional steps such as suturing procedures were necessary to close the corneal incision. The entire implantation was completed in an extremely short time period (typically less than $5 \mathrm{~min}$ ). As seen in figure 10, after one month, there was no device movement detectable in either of the rabbits implanted with the crossed anchor design illustrated in figure 6. Moreover, 

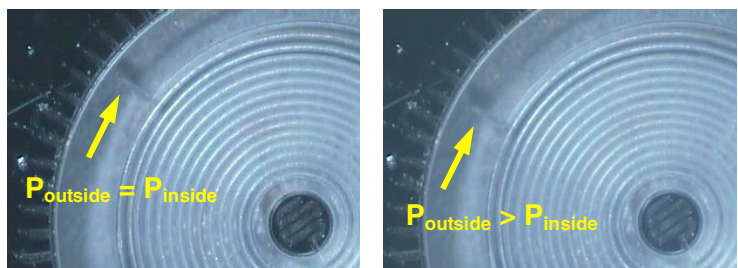

(a)
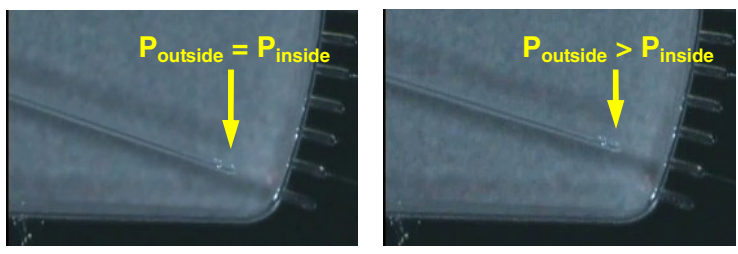

(b)
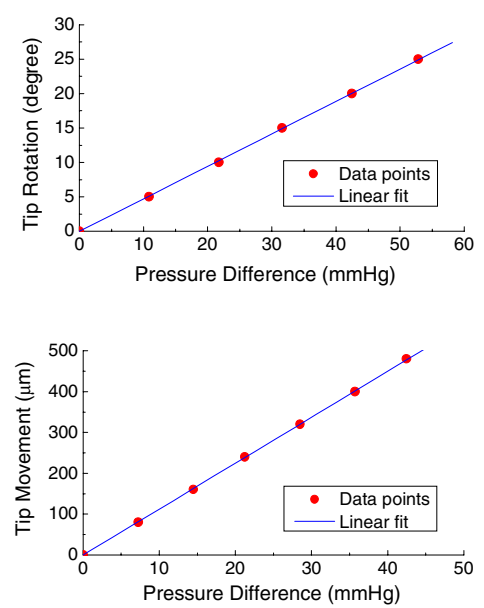

Figure 8. In vitro pressure response of the high-sensitivity IOP sensors in water: $(a)$ spiral-tube design; $(b)$ long-armed-tube design. The figure includes snapshots of tip movements before and after pressurization and associated measurement data.
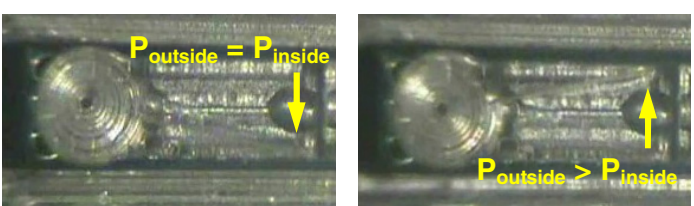

(a)

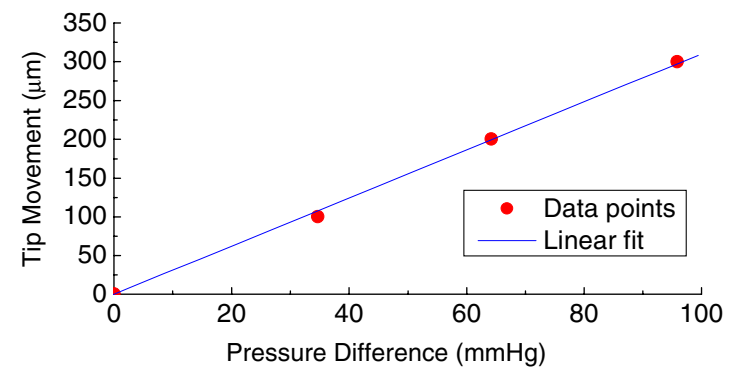

(b)

Figure 9. In vitro pressure response of the needle-implantable IOP sensors in water: $(a)$ snapshots of tip movement before and after pressurization and $(b)$ measurement data.

the follow-up fluorescein angiography over the month showed only very minimal leakage of blood in the iris, indicating that the anchors caused only minor trauma to the iris during implantation; over time, this leakage diminished.

\subsection{Ex vivo pressure sensor testing}

Implantation testing has been conducted in several enucleated porcine eyes to characterize the expected in vivo performance of the IOP sensors. For the high-sensitivity sensors, an angled blade incision and corneal suturing were performed to implant the devices due to their large physical dimensions. Testing showed that both the spiral-tube and long-armedtube designs successfully registered IOP variations in the intraocular environment under the external observation with a surgical microscope. Repeatable pressure responses of the sensors were confirmed by applying cyclic pressure variations. Figure 11 shows that a real-time, $8.72 \mu \mathrm{m} \mathrm{mmHg}^{-1}$ sensitivity was obtained in the long-armed-tube sensors, proving the microfabricated IOP sensors can achieve pressure sensing at the $\mathrm{mmHg}$ level in the intraocular environment. For the needleimplantable sensors, in spite of their minimally observable pressure responses due to low optical magnification of the scope, the devices were successfully implanted using a 19gauge needle as shown in figure 12.
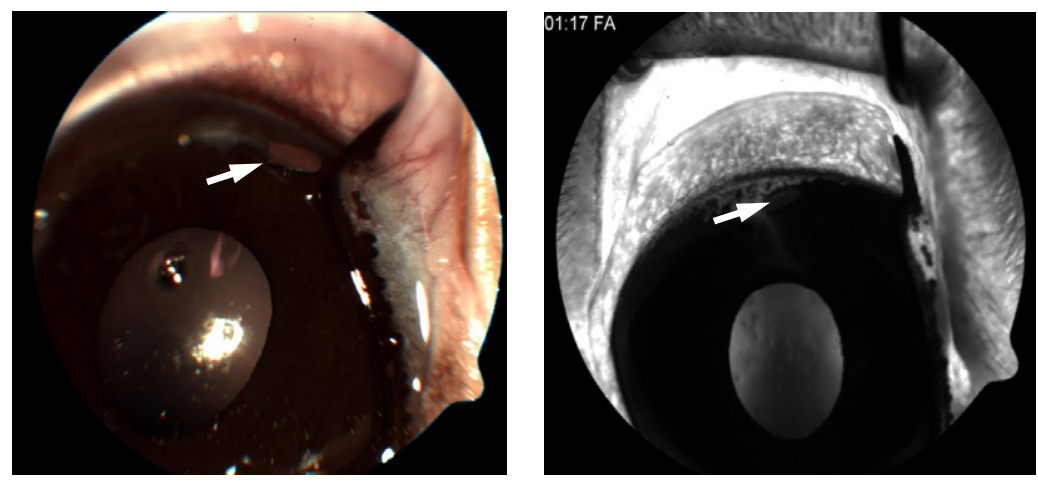

Figure 10. Typical in vivo anchoring test results with color fundus photograph (left) showing no device dislocation over one month and fluorescein angiogram (right) showing minimal blood leakage around device, which indicates little trauma to the iris. 


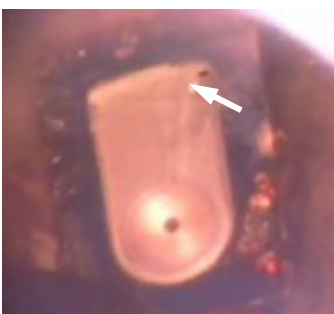

Low IOP (29 mmHg)

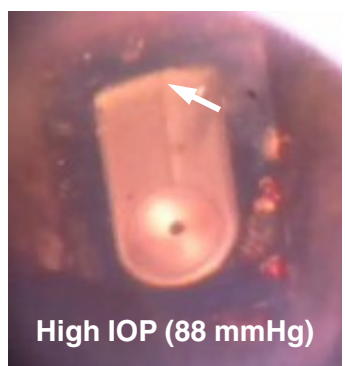

Figure 11. Ex vivo pressure response of the high-sensitivity long-armed-tube IOP sensor. The arrow denotes the position of the end pointing tip which changes with respect to the pressure variations in the intraocular environment.

\subsection{In vivo pressure sensor testing}

As another proof-of-concept demonstration, the needleimplantable pressure sensors were implanted in rabbit eyes and examined over time to evaluate their biostability. Over a 2 week follow-up period, one device still maintained its position as shown in figure 13 while another dislodged, possibly due to the large number of anchors in the middle of the device preventing good contact of the ends of the device with the iris. Other than that, after 2 weeks, the fluorescein angiography as shown in figure 13 confirmed only minimal leakage of blood around the device, again indicating only minor trauma to the iris. Devices that were completely coated in parylene showed almost no tissue encapsulation as compared to those with exposed silicon surfaces, which produced a discernable tissue response. Current work is underway to determine the optimal anchor number and geometry for more reliable device implantation in the intraocular environment, and to increase pressure sensitivity of the implant in a smaller form factor for more practical IOP measurements.
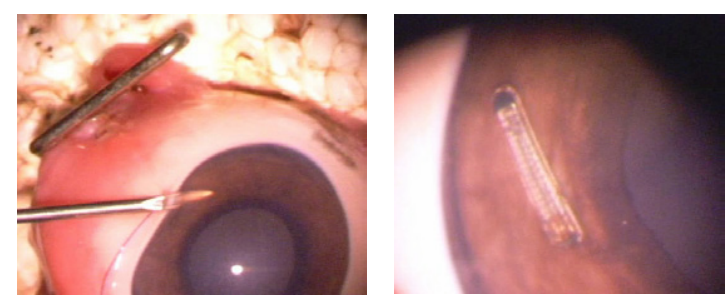

Figure 12. Ex vivo characterization of the needle-implantable IOP sensor: (left) device implantation through a needle incision; (right) device implanted inside the eye. The tissue anchors mechanically secure the device on the iris in the intraocular environment.
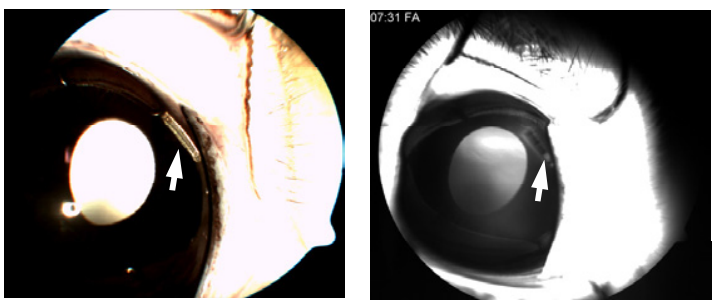

Figure 13. Pilot in vivo study in rabbit eyes with functional IOP sensors: (left) 1 week after implantation with no device dislocation; (right) 2 weeks after implantation with fluorescein angiogram showing minimal blood leakage.

\section{Conclusion}

An implantable, unpowered micromechanical parylene-based pressure sensor has been successfully developed as a part of a novel paradigm for intraocular pressure (IOP) sensing. Various geometric layouts for micromachined high-aspectratio flexible thin-walled tubes including a spiral-tube and a long-armed-tube design have been implemented to realize pressure sensing with different deformation trajectories. Depending on the target application, these devices are designed in a relatively large form factor to achieve high-sensitivity pressure responses, and in a small form factor to enable minimally invasive implantation through a needle. Monolithic fabrication and packaging of the microdevices have been successfully demonstrated. The micromachined IOP sensors were characterized in vitro, ex vivo, as well as in vivo. Testing results have yielded positive results toward practical IOP readouts at the $\mathrm{mmHg}$ level along with suture-less device anchoring after needle-based implantation. Long-term performance of the parylene spiral-tube pressure sensors is under study to verify the stability of their behavior in the intraocular environment. With the successful proof of concept, this new IOP sensor paradigm has a great potential of fulfilling unpowered, real-time, faithful and convenient IOP monitoring in glaucoma patients.

\section{Acknowledgments}

This work was supported in part by the Engineering Research Center Program of the National Science Foundation under NSF award number EEC-0310723 and by Bausch and Lomb. The authors especially wish to thank Mr Tun Min Soe for his work on the three-dimensional models shown in figures 1 and 2 and Mr Trevor Roper for his fabrication assistance.

\section{References}

[1] Sherwood M 2004 Ophthalmology ed M Yanoff and J S Duker (St. Louis: Mosby) pp 1413-73

[2] Cao H 2001 Minimally Invasive Medical Technology ed J G Webster (Philadelphia: Institute of Physics Publishing) pp 33-45

[3] Ethier C R, Johnson M and Ruberti J 2004 Ocular biomechanics and biotransport Annu. Rev. Biomed. Eng. 6 249-73

[4] Walter P 2002 Intraocular pressure sensor: where are we-where will we go? Graefes Arch. Clin. Exp. 240 335-6

[5] Collins C C 1967 Miniature passive pressure transensor for implanting in the eye IEEE Trans. Biomed. Eng. 14 74-83

[6] Rosengren L, Rangsten P, Bäcklund Y, Hök B, Svedbergh B and Selén G 1994 A system for passive implantable pressure sensors Sensors Actuators A 43 55-8

[7] Baldi A, Choi W and Ziaie B 2003 A self-resonant frequency-modulated micromachined passive pressure transensor IEEE Sensors 3 728-33

[8] Mokwa W and Schnakenberg U 2001 Micro-transponder systems for medical applications IEEE Trans. Instrum. Meas. 50 1551-5

[9] Chen P J, Rodger D C, Humayun M S and Tai Y C 2006 Unpowered spiral-tube parylene pressure sensor for intraocular pressure sensing Sensors Actuators A 127 276-82

[10] Clark R A and Reissner E 1950 Deformations and stresses in Bourdon tubes J. Appl. Phys. 21 1340-1 
[11] Meng E, Chen P J, Rodger D, Tai Y C and Humayun M 2005 Implantable parylene MEMS for glaucoma therapy Proc. Int. IEEE-EMBS Special Topic Conf. on Microtechnologies in Medicine and Biology (Oahu, HI, USA, 12-15 May 2005) pp 116-9

[12] Cookson Specialty Coating Systems, http://www.scscoatings.com/parylene_knowledge/ specifications.cfm

[13] Wolgemuth L 2000 Assessing the performance and suitability of parylene coating Med. Device Diagn. Ind. 22 42-9

[14] Schmidt E M, Mcintosh J S and Bak M J 1988 Long-term implants of parylene-C coated microelectrodes Med. Biol. Eng. Comput. 26 96-101

[15] Tooker A, Meng E, Erickson J, Tai Y C and Pine J 2005 Biocompatible parylene neurocages IEEE Eng. Med. Biol. 24 30-3

[16] Pang C, Musallam S, Rizzuto D, Ustun C, Tai Y C, Burdick J W and Andersen R A 2006 Vivo study of mechanical properties for the monolithic silicon probes with flexible parylene cables for neural prostheses Proc. Int. Conf. on Miniaturized Systems for Chemistry and Life Sciences (Tokyo, Japan, 5-9 November 2006) pp 702-4
[17] Ziegler D, Suzuki T and Takeuchi S 2006 Fabrication of flexible neural probes with built-in microfluidic channels by thermal bonding of parylene J. Microelectromech. Syst. 15 1477-82

[18] Li W, Rodger D C, Meng E, Weiland J D, Humayun M S and Tai Y C 2006 Flexible parylene packaged intraocular coil for retinal prostheses Proc. Int. IEEE-EMBS Special Topic Conf. on Microtechnologies in Medicine and Biology (Okinawa, Japan, 9-12 May 2006) pp 105-8

[19] Rodger D C, Weiland J D, Humayun M S and Tai Y C 2006 Scalable high lead-count parylene package for retinal prostheses Sensors Actuators B 117 107-14

[20] Chen P J, Shih C Y and Tai Y C 2006 Design, fabrication, and characterization of monolithic embedded parylene microchannels in silicon substrate Lab Chip 6 803-10

[21] van Drieënhuizen B P, Goosen J F L, French P J and Wolffenbuttel R F 1993 Comparison of techniques for measuring both compressive and tensile-stress in thin-films Sensors Actuators A 37-8 756-65

[22] Harder T A, Yao T J, He Q, Shih C Y and Tai Y C 2002 Residual stress in thin-film parylene C Proc. IEEE Int. Conf. on Micro Electro Mechanical Systems (Las Vegas, NV, USA, 22-24 January 2002) pp 435-8 